\title{
WIRKUNG UND REFLEXIONEN IN UNGARN
}

Es tut dem historischen Wert eines epochalen Ereignisses keinen Abbruch, wenn seine Ergebnisse und Lehren nicht sofort in Erscheinung treten, seine gesellschaftliche und ideelle Bedeutung wird aber nur noch mehr erhöht, wenn es, wie die Pariser Kommune, bereits in seinem Zeitalter als Wasserscheide aufgefasst wird.

Auch für die ungarische Bewegung lässt sich als nachhaltigstes Ergebnis die Verbreitung des internationalistischen Denkens bezeichnen. Einerseits: die Aufnahme der aus Frankreich geflüchteten Emigranten und deren Tätigkeit gaben der Bewegung der betreffenden Länder einen neuen Aufschwung, vergrösserten ihre Organisiertheit; andererseits: die Solidaritätsbewegungen machten eindeutiger, steigerten in den Arbeiterorganisationen das Gefühl der internationalen Zusammengehörigkeit. Das war eine grössere Geste, als sieben Jahre früher die offene Parteinahme für den polnischen Aufstand. 1864 basierte diese Stellungnahme auf einem zwar über Europa verbreiteten, inhaltlich aber nationalen Element, auf der revolutionären Unterstützung des Freiheitskampfes einer Nation. Die Kundgebungen im Jahre 1871 unterstützten hingegen offen den sozialen Inhalt und radikalen Charakter der Bewegungen selbst auf die Gefahr hin, dass dies in den einzelnen Ländern zum Bruch mit dem gemässigten Flügel führen würde, bzw. führen könnte.

Nichts ist natürlicher, als dass auch die Einschätzung der Ereignisse Unterschiede aufwies - der Einstellung der einzelnen Gruppen gemäss. Ein solcher Unterschied ist für den Widerhall in Ungarn besonders bezeichnend: während in der Lagebeurteilung der unabhängigen, bürgerlichen Linken - ähnlich, wie in der in Italien, welche mit sozialer und nationaler Erneuerung gleichzeitig geschwängert war - die nationalen, monarchiefeindlichen Züge der Pariser Kommune dominierten, trat die gegen die feudale Unterdrückung ankämpfende, noch am Anfang ihrer Organisationsarbeit befindliche Arbeiterbewegung für die Einführung der von der Kommune getroffenen sozialen Massnahmen in Ungarn ein. ${ }^{1}$

1 Ich berufe mich in meinem Aufsatz absichtlich zu Informationszwecken vor 
Von der Mitte des XIX. Jahrhunderts an hatte die sich in den westlichen Provinzen der Habsburgischen Monarchie entfaltende industrielle Revolution die früher zünftlerischen Industrieunternehmen oder Manufakturen in raschem Tempo erweitert; die Verbreitung der Dampfmaschine hatte nicht nur die Technik der Unternehmen revolutioniert, sondern auch die Dimensionen dieser Unternehmen stürmisch vergrössert. Trotz des Ausgleichs von 1867, der die Länder der Monarchie verfassungsmässig verband, war es immer merklicher zu verspüren, dass sich der Entwicklungsunterschied zwischen den österreichischen, bzw. ungarischen Territorien im Rahmen des gemeinsamen Zollgebiets immer mehr zuspitzte. Als Beispiel möchte ich nur erwähnen, dass in der Zeitspanne von 1867 bis 1879 von der österreichischen Regierung etwa das Vierzigfache dessen zur Unterstützung der Industrie verwendet wurde, was die ungarische Regierung aufwendete. ${ }^{1}$ Die Konkurrenz der österreichischen Grossindustrie zerrüttete das ungarische Kleingewerbe, die niedrigeren Preise der auf einer höheren Technik basierenden Massenproduktion förderten auch auf dem ungarischen Markt immer mehr die Entwicklung der Fabrikindustrie, führten aber andererseits, da sie hemmend auf die Entstehung der einheimischen, ungarischen Fabrikindustrie wirkten, notgedrungenerweise zur Konservierung der Kleinindustrie. Ungeachtet dessen, dass der verhältnismässig schnelle Ausbau des Verkehrs selbst die verlassensten Gegenden des Landes schnell in den Kreislauf des Wirtschaftslebens eingeschaltet und einen einheitlichen Markt geschaffen hatte und dass die grossindustrielle Produktion auf dem Gebiete der Nahrungsmittelversorgung, der Eisenerzeugung und des Maschinenbaus stürmisch an Raum gewann, hat sich infolge der eigenartigen ungarischen Wirtschafts- und Städteentwicklung die überwiegende Mehrheit der entstehenden Fabrikindustrie, und mit ihr ein Grossteil der ungarischen Industriearbeiterschaft in Budapest konzentriert. Ausserdem musste auch mit dem Vorhandensein einer bedeutenden Arbeitermasse gerechnet werden, die ihre Existenz in der Kleinindustrie zu behaupten imstande war. ${ }^{2}$

allem auf Schriften von ungarischen Verfassern; die in anderer Sprache erschienenen Studien sind ja den Forschern dieses Themas allgemein bekannt.

1 Vilmos Sándor, A tökés gazdálkodás kibontakozása Magyarországon (Die Entfaltung der kapitalistischen Wirtschaft in Ungarn), Kossuth-Verlag, Budapest 1954, S. 27.

2 Quellen zu den Angaben über Industrie und industrielle Bevölkerung sind: T. Iván Berend und György Ránki, Közép-Kelet-Európa gazdasági fejlödése a 19-20. században (Die wirtschaftliche Entwicklung Mittel- und Osteuropas im 19. und 20. Jahrhundert), Közgazdasági és Jogi Verlag, Budapest 1969, S. 32-43 (Kapitel „Aufschwung. Übergang zur modernen kapitalistischen Wirtschaft und die industrielle Revolution. Die demographischen Voraussetzungen der moder- 
1876 gab es in unserer Heimat 387636 Arbeiter, es arbeitete aber nur jeder Vierte von ihnen in Fabriken. Die Gesamtbevölkerungszahl der drei, im Gebiete der heutigen Hauptstadt Budapest befindlichen, damals noch selbständigen Städte Pesth, Buda (Ofen) und Obuda (Altofen) belief sich zur Zeit der 1870er Volkszählung auf 240476 Menschen (Ende 1872 bereits auf 300000). Zur selben Zeit gehörten zur Kategorie der damaligen Arbeiter (d.h. Hilfsarbeiter) 110726 Stadtbewohner. Als Fabrikarbeiter arbeiteten etwa 30 Tausend Menschen. Die sich hinter den Ziffern verbergende Proportion lässt sich am plastischsten durch einige vergleichende Angaben andeuten: in Paris zum Beispiel war allein die Zahl der weiblichen Fabrikarbeiter zweimal so gross, wie der Budapester Gesamtstand. In Osterreich war der Anteil der Bevölkerung im Vergleich zu der in Ungarn 42:58, der Anteil der industriellen Bevölkerung war 25:57 zugunsten Osterreichs. ${ }^{1}$

Nach dem Ausgleich, noch bis zur Jahrhundertwende, spielten bei der Gründung der Industrieunternehmen die deutschen und schweizerischen Facharbeiter eine ausschlaggebende Rolle. Der Anteil der ausländischen Facharbeiter erreichte in der Hauptstadt 24 Prozent; dazu kamen noch weitere, etwa 40 Prozent ausmachende heimische Arbeiter nichtungarischer Nationalität; aber auch der Grossteil der beschäftigten ungarischen Facharbeiter war bereits ausser Land gewesen. Demzufolge hatten die Kenntnis der europäischen Verhältnisse einerseits, die Zurückgebliebenheit der heimischen sozialen Zustände, die tragische Folgen nach sich ziehende Wohnungsnot, gepaart mit zahlreichen anderen weiterlebenden feudalen Unterdrückungsmethoden andererseits, die Klassengegensätze schon im voraus zugespitzt.

Die Folgen des Ausgleichs haben die Bedingungen der Arbeiterbewegung auch auf politischer Ebene mehrfach verändert. Das Régime des begüterten Adels und die örtliche Verwaltung hinderten die Geltendmachung der bürgerlich-städtischen Rechte mit allen Mitteln, ausserdem stand es ihnen in Ermangelung von Gesetzen, die die demokratischen Rechte gewährleisteten, frei, über das Schicksal einer jeden Versammlung oder Druckschrift auf dem Verwaltungswege oder polizeilich zu entscheiden. Letzten Endes hat aber all das im Vergleich zu

nen wirtschaftlichen Entwicklung"); Miklós Lackó, Ipari munkásságunk összetélelének alakulása, 1867-1949 (Gestaltung der Zusammensetzung unserer industriellen Arbeiterschaft, 1867-1949), Kossuth-Verlag 1961, S. 11-60.

1 Úber die Kleinindustrie: György Ránki, , A kisipar szerepe a magyar kapitalista fejlödésben" (Rolle der Kleinindustrie in der kapitalistischen Entwicklung Ungarns), in: Történelmi Szemle, Nr 2, S. 423-451. Die Angaben über PestBuda-Obuda (Pesth-Ofen-Altofen), d.h. über das heutige Budapest, lieferten die Jahrbücher des 1870 gegründeten Pester Städtischen Statistischen Amtes, vgl. Direktor des Pester Städtischen Statistischen Amtes Jozsef Körösi, A Pest vàrosi evkönyv (Jahrbuch der Stadt Pesth), Jg. I, 1873. 
den vergangenen Jahren, sogar Jahrzehnten doch günstige objektive Perspektiven eröffnet für die Organisationsarbeit der für ihren politisch-ökonomischen Interessenschutz in Kampf tretenden Arbeiter. ${ }^{1}$

Auch die subjektiven Voraussetzungen der Organisationsarbeit haben sich aus der Sicht der klassenkämpferischen Bewegung günstig gestaltet: infolge ihrer Zusammensetzung und der Erfahrungen ihrer Teilnehmer war die ungarische Arbeiterbewegung von Anfang an internationalistisch, ihre Blätter erschienen in zwei Sprachen, die Teilnehmer der Versammlungen ergriffen das Wort in ihrer Muttersprache. Die Tatsache, dass eine parteiähnliche politische Organisation - der Allgemeine Arbeiterverein (Altalános Munkásegylet) - verhältnismässig spät, erst 1868 gegründet wurde, erleichterte, dass in seinem Rahmen sofort die zeitgemässen Tendenzen die Oberhand behielten.

Obwohl sich in Ungarn am Anfang ein Lassalleanischer Einfluss Geltung verschafft hatte, wurde eine organisierte Verbindung nicht mit dem Allgemeinen Deutschen Arbeiterverein, sondern mit den dem Lager der I. Internationale angehörenden Eisenachern ausgebaut. Dies kann durch die Utbernahme einiger ihrer Programmpunkte im August 1869,2 sowie durch die Korrespondenz mit den Braunschweiger und Leipziger sozialistischen Leitern, durch die regelmässige Verbindung mit dem Londoner Generalrat der I. Internationale, mit ihrer Genfer Sektion und mit den der Internationale nahestehenden Wiener und Grazer Arbeiterorganisationen nachgewiesen werden. Solange keine selbständige ungarische Arbeiterpresse entstand, also bis 1870 , waren in Ungarn das Blatt der Genfer Sektion der I. Internationale, Der Vorbote, das Organ der Deutschen Sozialistischen Arbeiterpartei, Der Volksstaat, und das Blatt der Wiener Sozialisten, die Volksstimme, verbreitet gewesen. ${ }^{3}$

In der Erkenntnis der Bedeutung der heimatländischen Presse bei

1 Peter Hanàk, ,Skizzen über die ungarische Gesellschaft am Anfang des 20. Jahrhunderts”, in: Acta Historica, 1963, Nr 1-2 ; ,Hundred Years of Ausgleich”, in: The New Hungarian Quarterly, 1967, Nr 27.

2 Dezsö Nemes, Az Altalanos Munkasegylet törtenete 1868-1873 (Geschichte des Allgemeinen Arbeitervereins 1868-1873), Verlag Szikra, Budapest 1952, Kap. I; Edit Vincze, ,,Kampf um die Schaffung einer sozialistischen Massenpartei in Ungarn", in: Nouvelles Etudes Historiques, II.

3 Edit Vincze, ,A magyarorszagi es a nemet szocialista munkasmozgalom kapcsolatai a kivételes törvény elsö éveiben" (Beziehungen der ungarischen und deutschen sozialistischen Arbeiterbewegung in den ersten Jahren des Sozialistengesetzes), in: Párttörténeti Közlemények, 1971, Nr 1, und Edit Vincze, „A magyarorszagi munkáspart központi kölönye, az ,Altalános Munkàs Ujság' alapitàsanak 100. evfordulojara" (Zum 100. Jahrestag der Gründung des Zentralorgans der ungarischen Arbeiterpartei "Allgemeine Arbeiterzeitung"), in : Magyar Könyvszemle, Budapest 1970, S. 193-207. 
der Gründung einer selbständigen Arbeiterorganisation beschlossen im Frühjahr 1870 ,einige sozialistisch gesinnte Kopfarbeiter [...] ein sozialistisches Organ in ungarischer und deutscher Sprache ins Leben zu rufen", an Stelle des 1869 eingestellten Volksblattes mit Agitationscharakter Aranytrombita (Goldene Trompete). Das neue Blatt, die Allgemeine Arbeiterzeitung, war eine getreue Widerspiegelung der Zielsetzungen der Bewegung: es wirkte als Nachrichtenquelle und Organisator von Streiken und „preisregelnden” Aktionen. Es ist zu bemerken, dass es zwischen den beiden Varianten in deutscher, bzw. ungarischer Sprache auch einen grundsätzlichen Unterschied gab. Die ungarische Variante war weniger folgerichtig, da sie sich ihren Lesern angepasst hatte, die in ihrer Mehrzahl mit ihren Meistern in patriarchalem Verhältnis lebende Kleingewerbearbeiter waren. Obwohl das Blatt aus Mangel an finanziellen Mitteln bloss einige Monate existierte, brachte seine Organisations- und Propagandatätigkeit bleibende Resultate. Die gesellschaftliche und geistige Herkunft seiner Redakteure repräsentierte jene intellektuellen Schichten, die sich zur Sozialdemokratie hin orientierten. Sie waren: der aus Württemberg gebürtige Viktor Külföldi mit Universitätsbildung, der Korrektor des bürgerlich-liberalen Blattes Pester Lloyd Lajos Szvoboda und der bei den Kundgebungen des Jahres 1871 eine führende Rolle spielende achtzehnjährige Student der Technischen Hochschule Zsigmond Politzer. Alle drei wurden im ersten antikommunistischen Strafprozess unter der Anklage, mit der Kommune sympathisierende Bewegungen organisiert und sozialistische Lehren verbreitet zu haben, vor Gericht gestellt.

Im Vergleich $z u$ Berlin und Wien befand sich die Bewegung in Ofen-Pesth erst in ihrem Anfangsstadium. In jenen Städten, beide mit doppelt so grosser Bevölkerungszahl, betätigten sich 1871 in Berlin -ausser dem Allgemeinen Deutschen Arbeiterverein, dieser Landesorganisation mit fünfjährigen Erfahrungen - 51 Arbeitervereine: in Wien wirkten von 1869 an, ausser der 5000 Mitglieder zählenden Wiener Arbeiterbildungsanstalt, 20 Fachvereine. Zur selben Zeit existierte in Ofen-Pesth ein einziger Fachverein, der der Drucker. Er wurde 1870 unter dem Namen Munkásbiztositó Pénztár (Arbeiterversicherungskasse) gegründet, und hatte 1500 eingeschriebene Mitglieder. Aber im darauffolgenden Jahr begann der Prozess des Zusammenschlusses der Organisationen. Immer mehr Fachvereine schlossen sich dem Allgemeinen Arbeiterverein an. Die unter aktiver Mitwirkung des deutschen Sozialdemokraten August Rüdt und des österreichischen Sozialdemokraten Leopold Schäftner ins Leben gerufene Fachvereinssektion erfüllte eine wichtige Rolle bei den Kundgebungen des Jahres 1871. ${ }^{1}$

1 Tibor Erényi, A magyarorszagi szakszervezeti mozgalom kezdetei, 1867-1904 
Auf einem Irrtum oder aber auf der primitiven Leugnung der heimischen sozialen und politischen Umstände basierte der Bericht des Pester Stadthauptmanns vom 14. August 1871, der die Gründe der Wirkung der Pariser Kommune und den Hintergrund ,der Entstehung der Arbeiterfrage" in der internationalen Organisationsarbeit und im ausländischen, hauptsächlich durch österreichische Vermittlung nach Ungarn gelangten Einfluss suchte. ${ }^{1}$ Die Arbeitervereine, ihre politischen und wirtschaftlichen Aktionen waren nicht Ursachen der rapiden Organisationsentwicklung, sondern drückten jene sozialen und politischen Gegensätze aus, die damals im Lande vorherrschend waren. Die grosse internationale und ideelle Bedeutung der Pariser Kommune könnte durch nichts anderes besser nachgewiesen werden, als durch den Umstand, dass ihre Losungen ,in ungarischer Ubersetzung” in Ungarn die Verbreitung der sozialistischen Lehren, den Beginn der organisierten Kämpfe für Interessenschutz, die Erkenntnis der Notwendigkeit der Herausgestaltung einer selbständigen politischen Konzeption bedeuteten. Selbst übergangsweise betrachtete es keiner als Aufgabe, die staatlichen und ökonomischen Organisationsformen des Pariser Proletariats $\mathrm{zu}$ kopieren, die Losungen der Kommune wurden von niemandem unverändert wiederholt. Das unvergängliche Verdienst der Kommune besteht eben darin, dass ihre Wirkung gerade zur spezifisch nationalen Formulierung der gesellschaftlichen Probleme Schwung verlieh.

Informiertheit und Verbreitung der Arbeiterpresse blieben hinter denen der bürgerlichen Presse zurück, ${ }^{2}$ das nur von Februar bis Mitte April erscheinende Blatt Testvériség und sein deutsches Mitblatt Brüderlichkeit - die die Abänderung der Klassenstruktur der französischen Regierung anfangs von einer zunehmenden Teilnahme der Arbeiterklasse und ihrer Verbündeten erwarteten - haben aber die Bedeutung der Kommune trotzdem bald erkannt. Der am 26. März veröffentlichte, aus Paris eingelaufene Arbeiterbrief wurde unter dem Titel „Es lebe die Sozialdemokratische Republik” veröffentlicht. Der bekannte Leitartikler des Blattes, Viktor Külföldi, schrieb unter dem gleichen Titel einen Essai über die „Soziale Demokratie”, deren

(Anfänge der ungarländischen Gewerkschaftsbewegung, 1867-1904), Verlag Tancsics, Budapest 1962, S. 23-26 und 35-42.

1 Magyar Munkasmozgalom Történetének Vàlogatott Dokumentumai (Ausgewählte Dokumente der Geschichte der ungarischen Arbeiterbewegung, des weiteren: MMTVD), Verlag Szikra 1951, Band I, S. 179.

2 Für die bisher ausführlichste Bibliographie der zeitgenössischen Presse siehe: László Geréb, A párizsi kommün az egykoru magyar irodalomban (Die Pariser Kommune in der zeitgenössischen ungarischen Literatur), Fövárosi Szabó Ervin Könyvtár, Budapest 1951. 
Garanten er in einem Arbeiterstaat des Typs Kommune erblickte. Das grösste Interesse riefen aber in der heimischen Arbeiterbewegung und Presse zweifellos die auf den Arbeitslohn, auf die Regelung der Arbeitszeit bezüglichen, sowie gegen den Mietwucher gerichteten Massnahmen hervor, sie hatten nämlich den organischen Zusammenhang zwischen der Arbeitermacht und den sozialen Errungenschaften offenbar gemacht.

Im Mittelpunkt der Tätigkeit des Allgemeinen Arbeitervereins standen im Jahre 1871 die sozialistische Aufklärungspropaganda, die Organisierung von Versammlungen, die Erweiterung der Vereins zum Landesverein, und - im Rahmen der Interessenschutz-Tätigkeit - die Organisierung von verschiedenen Aktionen. Die Streiks standen vom März-April an bereits im Dienste der Verkürzung der täglich 13-14-stündigen Arbeitszeit und einer 25-prozentigen Erhöhung der durch Preisauftrieb der Verbrauchsartikel entwerteten Löhne. Ein Grossteil der Kundgebungen trug keinen spontanen Charakter, sie wurden vom Allgemeinen Arbeiterverein organisiert.

Die Wirkung der französischen Revolution bewusst und planmässig verwertend, die ständige polizeiliche Behelligung auf sich nehmend, gründeten die Mitglieder des Allgemeinen Arbeitervereins im Laufe von 1871 in nicht weniger als 14 Industrie- und Universitätsstädten Zweigvereine. „Allein die Arbeiter erkannten”, schreibt Géza Schulhof, der Jurist des Vereins und der spätere Chronist der Geschehnisse, „die Bedeutung der Pariser Ereignisse. Sie gerieten überall in Bewegung, und blickten mit Stolz auf ihre Pariser Brüder, die [...] dem Sozialismus eine neue, noch grössere Bedeutung verliehen." 1

Unsere Geschichtsliteratur hat die Ereignisse im Mai und Juni 1871 ausführlich bearbeitet. ${ }^{2}$ Sie befasste sich mit dem Streik vom 8 . Mai, an dem sich mehr als 3000 Arbeiter beteiligten und der ausser mit Lohnforderungen gegen die vor zwei Wochen erfolgte Verhaftung von 52 Vertrauensmännern geführt wurde. Im Verlaufe dieses Streiks drang ein Teil der Demonstranten sogar ins Parlament ein. Damit wurde den Machthabern zur Kenntnis gegeben, dass der ökonomische Kampf nunmehr einen politischen Charakter angenommen hatte. Im Streik kam auch die Überzeugung der Arbeiter zum Ausdruck, dass

1 Géza dombovari Schulhof, A munkasmozgalom keletkezése Magyarorszagon (Die Entstehung der Arbeiterbewegung in Ungarn), Verlag Kosmos, Budapest 1895, S. 27.

2 Tibor Erényi, „A magyarorszagi munkásmozgalom és az elsö Internacionale” (Die ungarische Arbeiterbewegung und die I. Internationale), in: Az I. Internacionale és Magyarorszag (Die I. Internationale und Ungarn), Kossuth-Verlag 1964. 
zu dauerhafter Lösung ausschliesslich in Gerichtsbarkeit und Verwaltung dürchzuführende Veränderungen führen könnten. ${ }^{1}$

Am 11. Juni berief der Allgemeine Arbeiterverein zum Andenken an die Pariser Kommune eine Versammlung ein. Ihre Teilnehmer trugen an ihren Hüten Trauerflor. Es wurde unter ihnen der aus 51 Punkten bestehende Beschluss des Internationalen Arbeiterverbandes Temple unique verteilt. Die Anwesenden „reizten”, nach Polizeibericht, „gegen die herrschenden Klassen der Gesellschaft, sowie gegen das die Arbeiter unterdrückende sogenannte Grosskapital oder Wucherkapital und gegen die gegenwärtige Regierung. Nach diesen Vorgängen führte die Polizei in den Lokalen des Vereins Haussuchungen durch, behelligte mehrere Tage hindurch mehrere hundert Mitglieder des Vereins, verhaftete 32 Führer, unter ihnen Andreas Scheu, der zur Organisierung des Vereins der Kleingewerbetreibenden nach Budapest gekommen war, ${ }^{2}$ sowie den in seiner Begleitung befindlichen Peschan,

1 Károly Farkas, Hauptbeauftragter der I. Internationale in Ungarn, einer der Hauptangeklagten des Abtrünnigkeitsprozess, später Mitglied der Ungarischen Allgemeinen Arbeiterpartei, berichtet in seinem Brief von 9.5.1871 an den Generalrat der I. Internationale: „Dreitausend Schneider und mit ihnen auch Arbeiter aus anderen Branchen besetzten das Abgeordnetenhaus und forderten die Freilassung der Gefangengenommenen. Die Führer der extremen Linken haben die Regierung wegen den Inhaftierungen scharf angegriffen und sie entlarvten in einer verspäteten Interpellation die gegen Raspe begangenen Schandtaten. [Friedrich Wilhelm Raspe, österreichischer Sozialdemokrat, beteiligte sich im Januar 1870 in der Organisation der Arbeiterbewegung von Pesth, im Februar desselben Jahres wurde er verhaftet. In dieser Angelegenheit und wegen der Auslieferung von Raspe interpellierten die beiden Abgeordneten Ernö Simonyi von der Unabhängigkeitspartei und der Führer der liberalen Opposition, Daniel Irányi.] Trotzdem ist das Vorgehen der extremen Linken nur eine Heuchelei, weil diese auf der einen Seite ebenso gute Bourgeois sind, wie die Konservativen [...] - auf der anderen Seite konnten wir in unseren direkten Kontakten zu ihnen feststellen, dass sie über die Arbeiterbewegung völlig falsche Vorstellungen haben. Diese meine Meinung vermochte ich nur sehr wenigen Leuten beizubringen." An dieser Stelle schildert Farkas den ganzen Verlauf des bewegten Tages vom 8.5.1871, dann zieht er folgende Konsequenzen: ,So stehen heute die Dinge. Durch den Ausstand werden wahrscheinlich keinerlei besondere Erfolge erzielt bis auf die Stärkung des Klassenbewusstseins. Die beiden Hauptvereine [der Allgemeine Arbeiterverein und der Arbeiterbildungsverein von Pest-Buda] versuchen nun zum drittenmal die Vereinigung, diesmal hoffentlich ernst und mit Erfolg. Die Fachvereine schliessen sich dem Allgemeinen Arbeiterverein an, um eine Massenorganisation zu bilden." Er schliesst seinen Brief folgendermassen: „Mit einem sozialistischen republikanischen Gruss und Handschlag." Veröffentlicht in MMTVD, I, S. 172-173, und in Források Budapest multjából (Dokumente aus der Geschichte von Budapest), Archiv der Hauptstadt Budapest 1971, Band I, Nr 144.

2 Herbert Steiner, „Die Gebrïder Scheu”, in: Archiv für Sozialgeschichte, Band VI-VII (1966-67), S. 489. 
die ,Mitglieder des Internationalen Zentrums', ausserdem die österreichischen Sozialdemokraten Schäftner, Kutil und Rüdt."1 Es dürfte gesetzmässig sein, dass die Schlussfolgerung am eindeutigsten gerade von Leo Frankel gezogen wurde, der vom August 1871 an im Generalrat der korrespondierende Sekretär für Österreich-Ungarn war. Er sagte auf der Londoner Konferenz der I. Internationale im September 1871 folgendes: „Die Arbeiterschaft Osterreichs hat besser als die Arbeiterschaft aller übrigen Länder begriffen, dass die Politik von den sozialen Fragen nicht zu trennen ist.” Er fügte noch hinzu: „Zum Siege der Revolution ist die Gründung einer selbständigen, den Parteien der begüterten Klassen gegenüberstehenden Arbeiterpartei unumgänglich notwendig." Gleichzeitig machte er auf die Schwere der bevorstehenden Kämpfe aufmerksam, indem er bewies, dass „die Regierungen [...] Angst davor [haben], dass sich die Arbeiterschaft in die Politik einmischen könnte". ${ }^{2}$ Frankel hielt die Gründung einer selbständigen Arbeiterpartei folgerichtig auf der Tagesordnung. Zur gleichen Zeit, als er auf der Sitzung des Generalrats vom 5. Juli 1872 über den gegen die ungarischen Arbeiterführer ausgeführten „Abtrünnigkeitsprozess" berichtete, kündigte er auch an, dass er einen Brief nach Buda geschrieben habe, in dem er die Arbeiter aufforderte, Arbeitssektionen ins Leben zu rufen. ${ }^{\mathbf{3}}$

Die Suche nach Verteidigungsmethoden gegen den gegen die Arbeiterbewegung gerichteten konzentrierten Angriff sowie das konsequente Auftreten der sogenannten bürgerlichen Linken im Parlament gegen den Regierungsterror warfen die für die sozialistische Bewegung so wichtige Frage der Verbündeten auf. Károly Farkas setzte sich zum Beispiel für die völlige Ablehnung eines Bündnisses mit den bürgerlichen Gruppen ein. ${ }^{4}$ Sein starres, verschlossenes, den Unterschied zwischen den einzelnen bürgerlichen Parteien ausser Acht lassendes Verhalten war unter den Arbeitern nicht volkstümlich. ${ }^{5}$ Dieses Verhalten wurde auch vom aufgeklärten bürgerlichen Publizisten Titusz

1 Von den nacheinander verhafteten 52 Personen wurde gegen 28 Anklage erhoben. Die Mehrzahl von ihnen ist erst Anfang 1872 auf freien Fuss gesetzt worden. MMTVD, I, S. 180.

2 Die Zitate stammen aus der vielseitig dokumentierten Biographie von Magda Aranyossi, Frankel Leo (Leo Frankel), Verlag Szikra, 1952, S. 69-70.

3 Weitere Einzelheiten aus dem Rechenschaftsbericht, sowie aus den bisher noch unveröffentlichten Briefen von Leo Frankel s. Janos Jemnitz und Edit Vincze, „Uj dokumentumok Frankel Leo munkassagarol" (Neue Dokumente über die Tätigkeit von Leo Frankel), in: Párttörténeti Közlemények, 1969, Tr 2, S. 121137.

4 S. oben S. 497, Anm. 1.

'Tibor Erényi, a.a.O., S. 330-331. 
Tóvölgyi einer Kritik unterzogen. ${ }^{1}$ Es ist wahr, Anfang des Jahres wirkte der angekündigte Zusammenschluss mit den „Unabhängigen”, ebenso wie das mit dem Nationalismus sympathisierende gemeinsame Memorandum hinderlich bei der Ausgestaltung einer selbständigen proletarischen Politik. Tatsache ist aber auch, dass die Erstarkung der unabhängig-deutschfeindlichen „äussersten Linken” die konterrevolutionäre deutschfreundliche aussenpolitische Orientierung der Regierung schwächte, während in der Innenpolitik die verschiedenen Richtungen der „Unabhängigen” der sozialistischen Agitation auch auf dem Lande Spielraum gewähren konnten.

Die Ereignisse im Mai-Juni 1871 haben die Frage des Verhältnisses der Parlamentarischen Parteien und der Arbeiterbewegung wieder aufgeworfen. Am 27. März interpellierte eine der führenden Persönlichkeiten der Unabhängigen, Dániel Irányi, unter Berufung auf die internationalen Verpflichtungen des politischen Asylrechts gegen die Haft von Wilhelm Friedrich Raspe und gegen seine etwaige Auslieferung an Osterreich; zwei Tage vor der Kundgebung, am 6. Mai, richtete der Abgeordnete Simonyi im Interesse der Freilassung der Arbeiter eine Interpellation an den Innenminister, und erbrachte den Beweis der Gesetzwidrigkeit ihrer Verhaftung. Es unterliegt keinem Zweifel, dass die Beurteilung der bürgerlichen Opposition in mehrerer Hinsicht einer Korrektur bedarf, und dies um so mehr, da die Pariser Kommune wenn auch infolge von denen der Arbeiterschaft völlig abweichenden Beweggründen - auch bei den bürgerlichen Kreisen der Intelligenz eine günstige Aufnahme fand.

Die Ereignisse der Kommune sind grösstenteils durch die bürgerliche Presse bekannt geworden. Dabei dürfte auch der Zufall eine Rolle gespielt haben; jener vierundzwanzigjährige Gábor Ugron nämlich, der aus dem revolutionären Paris vom April bis Oktober regelmässig berichtete, wollte ursprünglich zu Kossuth, um mit ihm ein Interview zu machen, und schloss sich später dem Heere Garibaldis an. Nach der Abrüstung dieser Armee ging er zusammen mit den Garibaldianern nach Paris. Seine Berichte und Feuilletons an Ort und Stelle sprechen zuerst im Tone eines sympathisierenden Beobachters und Plauderers. Später, mit dem Fortgang der Ereignisse, tritt er in seinen Beiträgen in den Zeitungen Ellenör, Gyors-Posta und Fövàrosi Lapok für die Kommune ein. Auch der Berichterstatter des Blattes Vasàrnapi Ujsàg (Sonntagszeitung) war nur zufällig in die Ereignisse hineingerissen

1 "A Commune Pesten" (Die Kommune in Pesth), in: Gyors Posta, 20. Juni 1871, und ,Szabadsag, mint a nép zàszlaja”, in: Gyors Posta, 25. Juni 1871. 
worden, als er auf dem Heimweg aus Amerika wegen Modemodellen in Paris abgestiegen war.

Die klerikalen und regierungsfeindlichen Blätter schwiegen die Pariser Bewegung tot, oder verleumdeten die französischen revolutionären Kräfte; das Blatt der Anhänger der Unabhängigkeit, Hon (Heimat), gerade so wie das Blatt von Deák, Pesti Napló, benutzten sie - begründet - als Argument für ihre Parteipolitik. Eine gewisse Befürchtung zeigte sich bei den um ihre Existenz bangenden Bürgern, dass der sich in Paris durchsetzende Radikalismus auch hier Anhänger finden könnte. Diesen Ansichten gab das Blatt Politikai Ujdonságok (Politische Neuigkeiten) Ausdruck, wenn es am 29. März erklärte, das Ziel sei erhaben, aber der Zeitpunkt für die Aktionen schlecht gewählt und die Methoden diskutabel. Wir haben der Wahrheit halber zu bemerken, dass dasselbe Blatt am 26. April nicht mehr den Aufstand tadelte, sondern eine Parallele zur Niederlage des ungarischen Freiheitskampfes zog und auf die tragischen Folge des Überhandnehmens der Reaktion aufmerksam machte.

Auf die liberalen Publizisten übte vor allem die Kontinuität der Pariser Kommune mit den revolutionären Traditionen und ihre moralische Kraft über der Gegenrevolution eine grosse Wirkung aus. Das Blatt Ország-Világ (Land und Welt) widmet in seiner Nummer vom 26. März den Ereignissen einen Leitartikel: „Nachdem das Volk überall aus der Rechnung gelassen oder vergessen wurde, kann es nicht Wunder nehmen, dass es die sich zeitweise bietenden Gelegenheiten ergreift. [...] Die niedrigen Schichten der Pariser Stadtbewohner vergassen nicht die Lehren, wenn sie auch die Bitterkeit vergassen, die sie über den Verlust der Verteidiger der Fahnen verspürten, die 1848 mit Kanonen von den Barrikaden heruntergeschossen wurden." Nándor Szederkényi erblickte in der Nummer vom 28. März des Blattes Magyar Ujság (Ungarische Zeitung) in der Märzumwälzung ein neues Gefecht des Kampfes zwischen den früheren Revolutionen und der Reaktion. Der Leitartikel der Politikai Ujdonságok vom 5. April würdigt unter dem Titel „Die Pariser Gemeinde”, dass „die Hauptstadt der Welt vielleicht jetzt den richtigen Weg findet, auf dem sie ihre führende Rolle wiederfinden könnte. [...] An der Spitze der Bewegung steht kein Lokalinteresse, sondern eine progressive gesellschaftliche Idee. [...] In diesem Land, wo Ende des vorangegangenen Jahrhunderts der Gedanke der gesellschaftlichen Gleichheit geboren wurde, $[\ldots]$ möchte man jetzt den grossen Druck des Kapitals und der grossen Vermögen erleichtern. Dies hingegen scheint wahrhaft eine Frage für die ganze Welt zu sein."

Die Behandlungsweise von Gefangenen und die Deportationen riefen auch bei Ugron Bestürzung hervor; im Blatt Ellenör schrieb er, 
auch er würde sie für Greuelmärchen halten, hätte er sie nicht persönlich gesehen. Ellenör schreibt spöttisch in seiner Auflage von 1.5. Juni: „Die Nationalversammlung ist den republikanischen Blättern im ganzen Lande nachgesetzt, das halbe Frankreich kann sie aber doch nicht erschiessen."

Selbst die Gegner der Pariser Kommune, die sich dessen bewusst waren, dass eine Abänderung der inneren Verhältnisse Ungarns abhängig ist von der Gestaltung der internationalen Kräfteverhältnisse, traten gegen das Uberhandnehmen der Reaktion auf. Ignác Helfy, ein der Regierung nahestehender Politiker des „Linkszentrums”, erklärte in seinem in der Nummer vom 31. Juni der Zeitung Magyar Ujság unter dem Titel „Politik der Rache" veröffentlichten Beitrag seine Freude über die Niederlage der Kommune, fügte aber sofort die Frage hinzu: „Wäre es möglich, sich auch nur vorzustellen, dass dieses ins Französische übersetzte Haynau-Regime imstande sein würde, die aufgepeitschten Parteileidenschaften zu besänftigen? [...] Und was tun hierauf die europäischen Kabinette? Sie schicken Begrüssungstelegramme, unter ihnen freilich vor allem das österreichungarische, d.h. das Wiener Kabinett [...] Henkern wollen wir nicht gratulieren, mögen sie Haynau oder Thiers heissen."

Die Vertreter der progressiven Öfentlichkeit sagten Paris, sprachen aber von Ofen-Pesth. Immer unverhüllter zogen sie eine Parallele zwischen dem Paris vom Jahre 1871 und der ungarischen republikanischen Bewegung von 1848-49, als die demokratische Volksbewegung von einer sich auf ausländische Waffen stützenden Monarchie abgelöst worden war. Unter dem Titel „Der Kampf der Idee” führt Szederkényi in der Magyar Ujság aus: ,Die Idee der Monarchie ist die Regierung, das Volk ist nur ein Anhängsel davon. Das Wesen der Republik ist: der Zusammenschluss des Volkes." Titusz Tóvölgyi, der sich vor allem mit der gesellschaftlichen Problematik der Kommune befasst, geht noch weiter: Auf die von ihm gestellte Frage: „Was ist also die Kommune?" erteilt er in der Zeitschrift $A$ szabadság, mint a nép zászlója (Die Freiheit als die Fahne des Volkes) - in der Nummer vom 7. Mai folgende Antwort: „Die Kommune bedeutet so viel, dass die DeákPartei in Bezug auf den Begriff der Freiheit um 300 Jahre zurücksteht." Er trat entschieden gegen den Zentralismus auf, der im Dienste der Willkürherrschaft stehe, und setzt sich für die Verwirklichung der „freien Gemeinden” ein.

Die hauptstädtischen bürgerlichen Kreise, die sich mit dem Gespinst der konservativ-zentralistischen Verwaltung besonders viel abplagten, meinten in der Pariser Kommune ein Musterbeispiel für das zukünftige Verwaltungssystem zu erblicken. Die Magyar Ujság stellt am 19. April die Monarchie der Freiheit entgegen, und weist darauf hin: 
Ihr Kampf geht um die Idee der Selbstverwaltung, das ist das grosse Postulat der Freiheit. Sonst kann die die Zentralmacht ergreifende Tyrannei die Freiheit des ganzen Volkes niedertreten. In einem Beitrag unter dem Titel „Kommune” (Magyar Ujság, 25. April) wirft Szederkényi die Frage historisch auf, indem er daran erinnert, dass selbst die grosse französische Revolution mehrmals von der Pariser Kommune gerettet wurde. In seinem angeführten Artikel verkündete Titusz Tóvölgyi den preussischen Waffen gegenüber den Zusammenschluss der freien Gemeinden.

Die Nachrichten, Kommentare, sowie die Lehren galten für Ungarn, sind aber zur gleichen Zeit im Zeichen der traditionellen Franzosenfreundschaft geschrieben worden. Die Sympathien wurden auch dadurch erhöht, dass sie von den Veränderungen in Frankreich das Ende der Hegemonie des preussischen Militarismus erhofften.

Die Stimmung der Öfentlichkeit kam vielleicht am besten darin zum Ausdruck, dass sich die für die breiten Schichten verfertigten "Witzblätter" ebenfalls oft mit dem Thema beschäftigten, und zwar auf Kosten der Bourgeoisie. Das Thema hat auch zahlreiche prominente Persönlichkeiten der ungarischen Lyrik mitgerissen, unter anderen die Dichter János Vajda und József Kiss. Die Lyrik zeigte den gesellschaftlichen Hintergrund dieser Geschehnisse nicht immer, forschte ihm nicht nach, umriss aber deren Stimmung schärfer und prägnanter, als jede andere literarische Gattung. Anstatt von Zitaten aus den einzelnen Gedichten mögen hier als Würdigung einige Sätze von György Lukács stehen, geschrieben über die Lyrik des XIX. Jahrhunderts: ,....] die Vorherrschaft der Lyrik unter den ungarischen Gattungen ist im voraus ein Abglanz der gesellschaftlichen Schwäche der radikalen Bewegungen. Es handelt sich nicht darum, dass die hochwertigen epischen und dramatischen Ausdrucksmöglichkeiten für die ungarische Entwicklung unbekannt wären. Sie kennt sie, sie kennt sie sogar sehr gut. Aber diejenigen ungarischen Schriftsteller, die sich berufen fühlten, die Geschicke unseres Volkes objektiv darzustellen, konnten gerade unter der Einwirkung ihrer Kenntnisse und Erfahrungen nicht zu den entschlossenen und kampffähigen Gegnern der feudalen Uberbleibsel und des sich mit ihm verschmelzenden Kapitalismus gehören. Der alleinstehende, trotz seines eventuellen Ruhmes doch auf sich selbst, auf die ausschliesslichen und unmittelbaren Offenbarungen seines eigenen Ichs angewiesene Lyriker hingegen schöpft die Grundtöne seines Pathos gerade aus dieser Situation."1

I György Lukács, „Bartok Béla” (Béla Bartok), in: Nagyvilag, Budapest 1970, Nr 9, S. 128. 
Ugron hat seinen Beitrag im Blatt Ellenör vom 15. August - auf dem Höhepunkt der Treibjagd gegen die Mitglieder der I. Internationale für ein Nachwort bestimmt: „Das soziale Úbel ist durch polizeiliches Verfahren nicht zu lösen." Die Zeitungen, wie es aus dem veröffentlichten Kommuniqué erhellt, ahnten schon, dass ein Nachwort, geschrieben am Rand der Niederlage, zugleich der Vorbote von neuen Zusammenstössen sein würde. Die ungarische Regierung hingegen hat die brutalsten Mittel der Unterdrückung schamlos in Anspruch genommen, und sie sogar weiterentwickelt.

Der Klassenkampf, der sich über ganz Europa ausbreitete, hatte klar gezeigt, dass die vorhandenen Mittel der Reaktion zur Unterdrückung der progressiven und Arbeiterbewegungen nicht mehr ausreichten, sie unterlagen im politischen Kampf. Diese schicksalsentscheidende Wandlung ist bekanntlich vor allem von Bismarck selbst erkannt worden, und zwar - nicht zufälligerweise - gerade nach der bekannten Parlamentsrede Bebels über das Pariser Ringen. ${ }^{1}$ Tatsache ist, dass Bismarck selbst vor der unmittelbaren Gewaltanwendung nicht zurückschreckte, aber schnellstens und weitestgehend bestrebt war, seine Massnahmen gesetzmässig zu untermauern, sie zu legitimieren. Nötigenfalls liess er eine ganze Reihe von diesbezüglichen Gesetzänderungen in Kraft treten. Alle diese Gesetze und Verordnungen sind in Mittel- und Osteuropa auch im Verlaufe der darauffolgenden Jahrzehnte in Kraft geblieben, sie funktionierten gegen die Arbeiterbewegung. ${ }^{2}$

Das zaristische Russland, die berüchtigt reaktionäre spanische Regierung, sowie die ihre inneren Gegner nur schwer im Zaum haltende Osterreich-Ungarische Monarchie haben sich als einander entsprechende Partner zur Schaffung einer neuen „Heiligen Allianz” erwiesen. An dieser Stelle möchten wir uns auf die zur Beurteilung der Stellungnahmen der ungarischen Regierung unerlässlichen Hinweise beschränken.

Es ist bekannt, dass Thiers Anfang Mai 1871 in Frankfurt von Bismarck freie Hand erhielt. Die schwankende und unentschlossene Wiener Regierung, die bis zur letzten Minute bestrebt war, als Gegengewicht zu Preussen die französische Regierung zu unterstützen, schlägt angesichts des Ausmasses der französischen Niederlage einerseits eine deutsch-österreichische Zusammenarbeit vor, andererseits reicht sie der Thiers-Regierung nach deren erster diplomatischer Geste im Zeichen der Solidarität gegen die Linke sofort ihre hilfreiche Hand:

1 Franz Mehring, Die Geschichte der deutschen Sozialdemokratie, Dietz Verlag 1919, Band V, S. 21-22.

2 Über die "Gleichschaltung" der Gerichte s. a.a.O., S. 22-24. 
am 25. Mai gab Beust dem österreichischen Geschäftsträger in Versailles den Auftrag, Thiers ihre diplomatische Anerkennung auszudrücken. Bereits am folgenden Tag, am 26. Mai, beauftragte Thiers den französischen Gesandten in Wien, er möge bei der österreich-ungarischen Regierung nachfragen, ob sie bereit wäre, die nach Osterreich geflüchteten Teilnehmer der Pariser Kommune auszuliefern. Der Gesandte leitete die Bitte am 2. Juni an Beust weiter, der sie nach den Regeln des Dualismus an das in inneren Angelegenheiten zuständige ungarische Ministerpräsidium weitergab. Beust deutet aber an: „Als ich im weiteren die Ehre habe, Eure Exzellenz höflichst zu ersuchen, ihre Ansichten über die Antwort an Marquis Bainville mir mitzuteilen, habe ich das Gefühl, dass ich meinerseits die Pflicht habe, jener über alle Zweifel stehenden Solidarität der Pflichten und Interessen Ausdruck zu verleihen, die jede Regierung den für den Todeskampf der Pariser Kommune bezeichnenden grauenhaften Exzessen gegenüber empfindet."

Die Regierung Andrássy erteilt ihre Antwort innerhalb einer Woche, und so erhält der Wiener französische Gesandte bereits am 18. Juni (nach 10 Tagen!) die österreichische Antwortnote: ,falls die französische Regierung um die Verhaftung einer solchen Person ersucht, die auf das Gebiet von Österreich-Ungarn geflüchtet ist, und die wegen der erwähnten Verbrechen steckbrieflich verfolgt wird, werden die zuständigen Behörden keine Schwierigkeiten verursachen in Bezug darauf, dass die verdächtigte Person sofort verhaftet und für den für das Einreichen des Auslieferungsantrags bestimmten Zeitraum in Haft gehalten werden soll. Der Auslieferungsantrag wird dann freilich vom zuständigen Gericht untersucht werden, das in jedem einzelnen Fall darüber $z u$ entscheiden hat, ob die betreffende Person an die französischen Behörden ausgeliefert wird."1

Es entstand also eine Zusammenarbeit zuerst mit der OsterreichUngarischen Monarchie - teils noch früher, als mit dem geographisch unmittelbar betroffenen Spanien. ${ }^{2}$ Inzwischen traf die Andrássy-

1 Istvan Dioszegi, Ausztria-Magyarorszàg es a francia-porosz hàboru (OsterreichUngarn und der französisch-preussische Krieg), Verlag der Ungarischen Akademie der Wissenschaften, Budapest 1965, S. 239-242. Alle Angaben über französisch-österreichische diplomatische Beziehungen sind diesem Buch entnommen. Der Reihenfolge nach: Jules Favre an Bainville, Paris 12.-26. Mai 1871, HHStA Wien, Min. des Äussern, Gesandschaftsarchiv Paris, Fasz. 148; Beust an Hoyos, Wien 25. Mai 1871, HHStA Wien, Min. des Äussern, Pol. Archiv IX, Frankreich, Fasz. 97; Abschrift einer Note an den Grafen Andrássy und Grafen Hohenwart, Wien 5. Juni 1871, und Beust an Bainville, Wien 18. Juni 1871, HHStA Wien, Min. des Äussern, Fasz. 148.

2 Zur selben Zeit war auch ein anderer Briefwechsel im Gange. Der Vater von Leo Frankel, ein hochangesehener, mit der k.u.k. Goldmedaille dekorierter 
Regierung Veranstaltungen, gegen die Arbeiterbewegung alles Bisherige übersteigende Massnahmen zu treffen. ${ }^{1}$ Die italienische Regierung hatte zwar den ihr von der deutschen Regierung nach der zwischen Bismarck und Gortschakow im Juli 1871 getroffenen Vereinbarung gestellten Antrag auf gegenseitige Information und Stellungnahme zustimmend zur Kenntnis genommen, näherte sich aber in ihrer Behutsamkeit eher der englischen Auffassung. ${ }^{2}$ Zwar war auch die englische Regierung nicht untätig geblieben: am 10. Juli erbat sie Informationen über die Tätigkeit der Internationale in ÖsterreichUngarn, ihre Massnahmen waren aber viel weniger einschneidend als die der ungarischen Regierung. Von der Osterreich-Ungarischen Monarchie jedoch ist der Antrag Bismarcks mit der grössten Bereitwilligkeit aufgenommen worden. ${ }^{3}$

Bismarck ,gewann den Grafen Beust bereits im Sommer 1871 für die Bewegung gegen die revolutionäre Assoziation. Gortschakow wollte sie ebenfalls mit Feuer und Schwert ausrotten, da er sich vor einer Uberpflanzung der sozialen Lehren der Internationale in russischen Boden fürchtete. Ähnlich wie Bismarck und Gortschakow, verschloss sich auch Andrássy nicht der Erkenntnis, dass gemeinsam festzulegende energische Massnahmen einzuführen wären gegen die für jeden Staat gleicherweise gefährlichen Tendenzen der ,ligue anti-

Wundartzt in Buda bat um die Intervention des Rates von Buda, um Nachrichten über seine in Paris lebenden Söhne erhalten zu können. ,I Ich ersuche höflichst den hohen Rat, möge er die hohe königliche ungarische Regierung zur Aufklärung dessen veranlassen, ob mein Sohn, Leo Frankel, von Beruf Goldschmied und schon seit Jahren in Paris wohnhaft, wirklich fusiliert wurde, wie darüber die öffentlich erscheinende Presse berichtete? und ob mein älterer Sohn Ingomar Frankel, Portraitmaler, der auch schon seit drei Jahren in Paris lebt und die Stadt weder während der Belagerung noch zur Zeit des bewaffneten Aufstandes verlassen hat, noch lebt, oder wenn nicht mehr, wie er umgekommen ist." Zwei Wochen nach dem Beschluss der ungarischen Regierung über die Auslieferung der Flüchtlinge, am 28. Juli, erhielt der Rat von Buda die Zuschrift Nr 1908 des Innenministeriums, mit der Mitteilung: ,Ingomar Frankel, der auf der Pariser k.u.k. österreichisch-ungarischen Botschaft angehört wurde, hat sich dahingehend geäussert, dass er seinen Vater sowohl über sein eigenes wie auch über seines Bruders Schicksal unmittelbar in Kenntnis bringen wird. Über das Verbleiben seines Bruders hat er sich nicht geäussert. Auf Grund dessen und anderer ihr zugekommenen Informationen vermutet die obengenannte Botschaft, dass Leo Frankel, ehemaliges Mitglied der Commune, sich noch immer in Paris versteckt hält." Archiv der Hauptstadt Budapest, Archiv von Buda, Aktenstücke des Rates 1884/871. Beide Aktenstücke sind veröffentlicht in Források Budlapest multjából, Band I, Nr 145 und 146.

1 Tibor Erényi, a.a.O., S. 336.

2 Federico Chabod, Storia della politica estera italiana dal 1870 al 1890, Band I: Le premesse, Bari 1951, S. 428.

3 Ferenc Pecze, , Die Staatstheorie des Dualismus”, in: Studia Iuristica, Nr 48. 
sociale'. $[\ldots]$ Und wenn auch in dieser Richtung in Berlin aller Wahrscheinlichkeit nach kein schriftliches Abkommen zustande gekommen war, gelangten sie mündlich vermutlich doch zu einer einhelligen und einheitlichen Vereinbarung gegen das Bündnis, welches die auf monarchistischer Grundlage stehende Gesellschaft in die Luft sprengen wollte."1

Am 9. Februar 1872 lief das „Mahnschreiben" des spanischen Aussenministeriums ein, welches zur unermüdlichen Fortsetzung des Kampfes gegen die I. Internationale aufrief. ${ }^{2}$ Das Aussenministerium der Monarchie teilt dies der ungarischen Regierung mit, die bereits auf ihrem Ministerrat vom 21. Mai die zu ergreifenden Massnahmen erörtert. ${ }^{3}$ Dieser Ministerrat fasste einen Beschluss über die Begrenzung des Vereinigungsrechts und des Streikrechts und über die Einengung der Offentlichkeit der bereits bestehenden Organisationen. Er schenkte ausserdem seine volle Aufmerksamkeit der Unterstützung und dem gesetzlichen Schutz der gegen die Arbeiterrechte gerichteten Organisationsarbeit. Er trat z.B. dafür ein, dass „im Kreise der Besitzenden” die Organisationsarbeit zu ihrem eigenen Schutz zu verstärken wäre; dass der gesetzliche Schutz der Streikbrecher auszuarbeiten wäre; dass schliesslich, ohne die früheren Gesetze in Bezug auf die Vereinigung und den Arbeitsplatzwechsel ausser Kraft zu setzen, ihre Auslegung zu modifizieren wäre. ${ }^{4}$ Auch die österreichisch-ungarisch-preussische „Expertenverhandlung" vom 7. bis 29. November 1872, an der dann ungarischerseits Lajos Jekelfalussy, der Chef der Polizeiabteilung des Innenministeriums, teilnahm, wirft bereits ihre Schatten voraus. Die Regierung schrieb auch die bei den Verhandlungen zu befolgende Richtlinie vor, indem sie festlegte: die Gesetze sind derart abzuändern, dass jede landweite Organisationsarbeit verhindert und alle diesbezüglichen Initiativen lokalisiert werden können. Als eine Merkwürdigkeit sei hier vermerkt, dass zwischen dem Deutschen Bund und

1 Ede Wertheimer von Monor, Gr. Andrássy Gyula élete és kora (Leben und Zeit von Graf Gyula Andrássy), Verlag der Ung. Akademie der Wissenschaften, Budapest 1913, Band II, S. 94.

2 Vgl, den Text des Schreibens: Dok. II im Anhang zum Artikel von Carole Witzig, oben S. $219 \mathrm{ff}$.

3 ,Der Ministerrat hat festgestellt, dass die Auslieferung von politischen Verbrechern" von den internationalen Verträgen untersagt wird. Der Ministerrat ist aber bestrebt, sich über diese Verbote hinwegzusetzen, , da es nicht zu leugnen ist, dass die Anhänger der Pariser Kommune solche Ruchlosigkeiten verübten, die unter dem Mantel von politischen Parteibestrebungen gar nicht gedeckt werden können." Langenau an Andrássy, März 1872, und Andrássy an Langenau, 11. April 1872. MMTVD, I, S. 245; Ede Wertheimer von Monor, ,Zur Geschichte der ,Internationale"', in: Pester Lloyd, 26. August 1908.

4 MMTVD, I, S. 246-249. 
Österreich-Ungarn bereits 1836 und 1854 Abkommen über die gegenseitige Auslieferung der gewöhnlichen und der mit ihnen gleichgestellten politischen Verbrecher zustande gekommen waren. 1920 hat sich anlässlich des Prozesses gegen die Volkskommissare und später bei dem Auslieferungantrag gegen Béla Kun auch die ungarische HorthyRegierung - unter anderem - auf dieses Abkommen berufen. ${ }^{1}$

In Ermangelung der notwendigen gesetzlichen Basis vermochte auch die ungarische Regierung - trotz der Verhaftungen und trotz ihrer diesbezüglichen Entscheidung - keine schnellen Massnahmen zu ergreifen. Das „Linkszentrum” brachte seine Interpellationen serienweise gegen die Ausweisung von ausländischen und die Gefangenhaltung von ungarischen Staatsbürgern ein. Die Antworten des Innenministeriums und des Justizministeriums liessen zwei Monate auf sich warten, bis sie endlich erklärten: „Den beschlagnahmten Dokumenten nach standen mehrere angeklagte Mitglieder des Budapester Arbeitervereins in Korrespondenz mit dem Internationalen Bund in London und Genf, mit New-York, Bruxelles, Zürich, Kairo, Berlin, Braunschweig, Hamburg, Eisenach, Reichenberg, Graz, Wiener-Neustadt, beziehungsweise mit den dort tätigen internationalen Agitatoren, in Ungarn hingegen gründeten sie Filialvereine zur Ausführung ihrer Pläne."2

Aufgrund der gegen die 28 inhaftierten Arbeiter durchgeführten Untersuchung lassen sich die in Untersuchungshaft befindlichen Arbeiter in drei Kategorien einstufen: zur ersten Klasse gehören diejenigen, die sich als Zentralmitglieder der Genfer Internationale zur Annahme der internationalen Grundsätze verpflichtet haben und ein bei den Haussuchungen vorgefundenes Aufnahme-Dokument besitzen. Von ihnen gab es fünf, einer davon ist inzwischen laut ärztlichem Befund an einer vorgeschrittenen Lungentuberkulose gestorben. Zur zweiten Klasse gehören diejenigen, denen dokumentarisch zwar nicht nachgewiesen werden konnte, dass sie Zentralmitglieder des Internationalen Bundes wären, die aber mit den Verbreitern der Grundsätze des Zentralbundes teils in Korrespondenz standen, teils sich aktiv bei der Verbreitung dieser Grundsätze betätigten. Zur dritten Klasse können schliesslich diejenigen gezählt werden, die als Mitglieder des Vereins an den Kundgebungen und Versammlungen teilgenommen haben, und die von den Leitern zur Erreichung ihrer Ziele als Mittel gebraucht worden sind. Diese waren 15 an der Zahl, und die Verwaltung der königlichen Angelegenheiten (die Staatsanwaltschaft) hat sofort nach der Beendigung der Untersuchung ihre Freilassung angeordnet.

1 Ges. Gaus an den Reichsminister, Berlin 20. Juli 1920, Auswärtiges Amt, Politisches Archiv, Bomn, Politische Abteilung, Bela Kun Film Nr K 284508-10.

2 MMTVD, I, S. 191-192. 
Momentan werden also noch 12 Arbeiter gefangengehalten, gegen sie wird aufgrund der Gesetzesartikel VII./1715., IX./1723. und LVI./ 1790-91. (über Majestätsbeleidigung, Abtrünnigkeitsverbrechen und Aufruhr) die Anklageschrift bei der Königlichen Tafel demnächst eingereicht werden. Die Königliche Tafel als zuständiges Gericht wird dann die Aufgabe haben, gleichzeitig auch über die Frage zu entscheiden, ob einige von diesen Gefangenen bis zur endgültigen Urteilsfällung auf freien Fuss gesetzt werden könnten, und, wenn ja, wer von ihnen. ${ }^{1}$

Die Verhandlung in Sache des ungarischen ,Abtrünnigkeitsprozesses” wurde dann - nach langwierigen Vorbereitungen - in der Zeit vom 23. bis 29. April 1872 abgehalten, und das Urteil ist am 1. Mai verkündet worden. Man war gezwungen, alle 28 Angeklagten freizusprechen, ${ }^{2}$ da man in Ungarn in politischen Prozessen diesen Typs noch keine Erfahrung hatte, obwohl es offenkundig ist, dass man - wie bereits früher angedeutet - im Zeichen der „Rechtskontinuität” selbst vor Anwendung jahrhundertealter Gesetze nicht zurückschreckte. Von 1872 an bereitete dann die Regierung all jene Gesetze und Verordnungen in beschleunigtem Tempo vor, die später bei den brutalen Massnahmen gegen die Arbeiterbewegung anwendbar waren.

Auch hier wollte man aber die „Rechtskontinuität” - teils mit dem königlich-ungarischen Recht, teils mit der Gesetzgebung des Freiheitskampfes - aufrechterhalten, um die neuen Gesetze für breitere Schichten der Offentlichkeit annehmbar zu machen. Der Schöpfer des neuen Gesetzes, Károly Csemegi, und der im Prozess gegen Leo Frankel als Richter fungierende Lörinc Tóth waren selbst Teilnehmer des Freiheitskampfes, die auf die Basis der Gesetzmässigkeit hinüberwechselten, geleitet zunächst durch persönliche Interessen, später auch durch

1 Béla „Sarlos, Csemegi Károly és a kiegyezés" (Károly Csemegi und der Ausgleich), in: Történelmi Szemle, 1968, Nr 1-2.

2 Der ungarische Ministerrat, der sich zweimal mit den verschiedenen gegen die Führer der Arbeiterbewegung eingeleiteten Prozessen beschäftigte, stellte unter anderem fest, dass diese Arbeiterführer direkte Kontakte zu den verschiedenen Zentren der damaligen europäischen Arbeiterbewegung hatten, z.B. zu Beauftragten der Internationale in London und in Genf. Ier Justizminister hat dahingehende Bedenken geäussert, ob eine Anklage damit ausreichend begründet sei, dass die ungarischen Arbeiterführer Aufträge von der Internationale erhalten haben, wobei der Beweis der Ausführung dieser Aufträge nicht zu bewerkstelligen war. Ein Prozess wurde schon deshalb in Frage gestellt, weil die in Wien verurteilten Arbeiterführer vom Kaiser begnadigt wurden. Der Ministerpräsident des ungarischen Kabinetts teilte diese Bedenken seines Justizministers nicht. Die Mehrheit des Kabinetts schloss sich der Meinung der Ministerpräsidenten an. Diese Stellungnahme der Regierung determinierte den eingeleiteten Prozess insofern als der Angeklagte Politzer zu sechs Monaten Gefängnis verurteilt wurde. Die Mehrheit der Angeklagten kam nur deshalb unbestraft davon, weil die von der Anklagebehörde zusammengetragenen Beweise zur Verurteilung nicht ausreichten. Wortlaut des Protokolls s. MMTVD, I, S. 200-204 und 228-234. 
eine prinzipiell antirevolutionäre Einstellung. ${ }^{1}$ Csemegi nahm z.B. von 1867 an jede Gelegenheit wahr, um den revolutionären Charakter des österreichischen Standpunktes nachzuweisen, und bei jeder österreichischen Argumentation und Thesis auf deren revolutionären Ursprung hinzuweisen.

Ausserlich gesehen ist der im ungarischen Recht nach 1867 vorherrschende Aspekt vielleicht ,national”, es verwirft aber die Enzyklopädisten und die Vertreter des Absolutismus gleicherweise darum, weil jene die Vergangenheit für ,aller Rechte verlustig” erklärten, und durch die neuen Gesetze einen „künstlichen Mechanismus” schaffen wollten. Der ungarische Standpunkt besteht hingegen darin, dass die Verfassung des Staates nicht das Eigentum einer einzigen Generation sei, über die diese Generation frei verfügen dürfe, sondern dass sie das Eigentum des Staates selbst sei. Der Staat wiederum sei ein moralisches Ganzes, welches „sich als eine Person kundtut”, und dessen jeweilige Hüter die Prinzipien des Staates nach den Bedürfnissen des Fortschritts weiterentwickelten. Die konservative, im Grunde genommen antirevolutionäre ungarische Rechtsauffassung ging sogar noch weiter: nach dem Ausgleich stellte es sich heraus, dass das Recht über keine selbständige Existenz verfügt. Gegenüber dem vom Jahre 1848 ist der Staat antidemokratisch geworden, und von dieser Zeit an ist selbst jener Teil des Gesetzes, welcher scheinbar unverändert im Gewande von 1848 auftritt, Handlanger dieses neuen Beschlusses geworden. Durch den $\S 12$. der Regierungsverordnung 64./1867., eines vertraulich hantierten Statuts, ist - abweichend von 1848 - das Vorsanktionierungsrecht des Königs eingeführt worden. Die Verordnung 307./ Präs. 1867. hat zwar das Pressegesetz von 1848 formell nicht abgeändert, schaffte aber die Anklage vor der Geschworenenbank ab und veränderte die Struktur des Deákschen Schwurgerichts von 1848. Der Gesetzesartikel XLIV./1848. wich in der Beschränkung der Nationalitäten auch in der Praxis von der Stellungnahme der Nationalversammlung von $1861 \mathrm{ab}^{2}$ Die Anwendung des Strafgesetzbuches ist mit

1 Uber den Lebenslauf von Lörinc Tóth und Károly Csemegi siehe die romanhafte Biographie der führenden Gestalt des Justizwesens des Freiheitskampfes 1848-49, Sebö Vukovics, geschrieben von Béla Sarlós, Deák és Vukovics. Két igazságügyminiszter (Zwei Justizminister, Deák und Vukovics), Verlag der Ungarischen Akademie der Wissenschaften 1970, Nachwort, S. 102-104. Ausserdem: Erika Rév, A népbiztosok pere (Der Prozess der Volkskommissare), Kossuth-Verlag 1969, S. 14-20.

2 Zum Beweis der obigen Feststellung dienen die vorhergegangenen Ereignisse des Presseprozesses gegen Leo Frankel und auch das Urteil. Weil es nach den schon bekannten französischen Beschlüssen nicht mehr möglich war, Leo Frankel abzuurteilen und ihn auf diesem Wege aus der Bewegung radikal zu entfernen, kamen die verschiedenen Schikanen gegen ihn auf die Tagesordnung. 
einer von der von 1848 völlig abweichenden Praxis in die Hände von ernannten Richtern gelegt worden.

Die Angst vor der Arbeiterbewegung, die gegen sie getroffenen Massnahmen haben sich auf alle Gebiete der Gerichtsbarkeit erstreckt. Der zunehmende Terror zeigt sich vor allem in den Presseprozessen, da sich der politische Gegner meistens dort offenbart und sich zu Retor-

1876 wurde er in der Erwartung eines französischen Auslieferungsantrags inhaftiert und erst nach dreieinhalb Monaten und gegen eine Kaution von 5000 Kronen wieder auf freien Fuss gesetzt. $\mathrm{Zu}$ einer Aburteilung wegen seiner Tätigkeit in Ungarn gab es keine ausreichenden Gründe, und - wie bekannt hat die französische Regierung seine Auslieferung nicht beantragt. (Den nach der Untersuchungshaft geschriebenen Brief von Frankel publizierte János Jemnitz.) Deshalb versuchte die Polizei die Ausdehnung der polizeilichen Pressezensur auf die von Frankel zu erwartenden Artikel. Frankel, der diese behördlichen Versuche durchschaute, informierte am 20. Januar 1878 schon zum zweitenmal die Budapester Polizeihauptmannschaft über den Inhalt seiner Rede, die er am selben Tag auf einer Grosskundgebung hielt.

,Abgesehen davon, dass wir keine Zensur in Ungarn besitzen, die mich verpflichten würde, die Gegenstände, die ich mündlich oder schriftlich zu behandeln wünsche, erst der betreffenden Behörde zu unterbreiten, abgesehen davon, dass bei allen öffentlichen Versammlungen behördliche Vertreter anwesend sind, die darauf $z u$ achten haben, ob der eine oder andere Redner von der Tagesordnung abweicht oder in einer gegen die Landesgesetze verstossenden Weise auftritt, und in diesem Fall von der kompetenten Behörde zur Rechenschaft zu ziehen sind und eventuellen Falles nach unseren Gesetzen auch bestraft werden können, abgesehen davon ist es mir unmöglich, den Vortrag, den ich heute nachmittag zu halten gesonnen bin, schriftlich mitzuteilen, da ich zu meinen Vorträgen kein Konzept zu machen pflege, sondern nur einzelne Notizen. Das Thema, das ich behandeln will, ist meines Erachtens klar genug ausgesprochen in der der Oberstadthauptmannschaft vorgelegten Anzeige. Ich will dessenungeachtet, um alle diese Missdeutungen zu beheben, hiermit darlegen, dass ich unter Freiheit der Wissenschaft und ihver Lehre verstehe, dass alle Wissenschaften, ob sie nun den einen oder anderen in seiner Vorstellung, seinem Glauben, seinen bisherigen Ansichten stören, in keine Schranken beengt werden dürfen, ihre Lehre frei sein muss, weil sonst der Trieb wissenschaftlicher Erkenntnis selbst erstickt wird. Ohne die Freiheit der wissenschaftlichen Erkenntnis, die gerade eine Errungenschaft der modernen Zeit ist, gibt es kein geistiges Leben, sondern nur Stagnation, Versumpfung. Ohne Freiheit der Wissenschaft und ihrer Lehre gäbe es nur Aberglaube, Vorurteil, geistige Nacht. [...] Wie stünde es mit der Geologie, wenn sie sich in ihren Forschungen über das Alter der Erde an die biblischen Aussprüche zu halten hätte, wie mit der Medizin [...], wenn sie alle Krankheiten, alle Seuchen als ,Gottesstrafen' betrachten müsste, die nur durch Gebete zu beseitigen seien. [...] Wenn es auch schon verboten ist, sozialistische Agitationen zu betreiben, wie dies in der letzten Verordnung des $\mathrm{k}$. Ministeriums des Innern lautet, so ist es gegenwärtig wenigstens noch nicht verboten, die wissenschaftliche Erkenntnis im Volke zu fördern, und ich hoffe demnach mit $Z$ uversicht, dass die [...] angekündigte Versammlung von der Oberstadthauptmannschaft zur g. Kenntnis genommen werden wird." Archiv der Hauptstadt Budapest, Archiv der Oberstadthauptmannschaft, veröffentlicht in Források Budapest multjából, Band II, Nr 94. 
sionen seitens der Regierung meistens unter dem Vorwand von Pressedelikten Gelegenheit bietet. ${ }^{1}$ Wir dürfen nicht vergessen, dass (mit Ausnahme der Kompetenz der Königlichen Tafel) die nach dem Ausgleich ernannten Staatsanwälte und Richter zuerst bei den erstinstanzlichen schwurgerichtlichen Pressevergehen mitwirkten, dass der Einfluss der Munizipien auf die Aufstellung des staatsanwaltlichen und richterlichen Apparats zuerst bei den Presseprozessen aufhörte. ${ }^{2}$

So ist der Terror gegen die aufstrebende Arbeiterbewegung in der Hand der "Juristennation", genauer gesagt, in der Hand der Regierung eine Waffe zur Unterdrückung jeglicher progressiven und oppositionellen Bewegung geworden.

Aus dem Ungarischen übersetzt

1 Béla Sarlos, A sajtoszabadság és eljárasi biztositékainak fö vonàsai (Hauptzüge der Pressefreiheit und ihrer verfahrensmässigen Garantien) [Jogtörteneti Tanulmanyok (Rechtsgeschichtliche Studien), III], Közgazdasàgi és Jogi Kiado, Budapest 1968.

${ }^{2}$ Noch im Laufe desselben Jahres 1878 ist in der von Leo Frankel redigierten deutschsprachigen Arbeiterzeitung Arbeiter Wochen Chronik ein Wiener Flugblatt veröffentlicht worden, welches in der dortigen Presse schon früher veröffentlicht worden war. Dasselbe Flugblatt ist in ungarischer Ubersetzung auch im fortschrittlich-bürgerlichen Blatt Függetlenség (Unabhängigkeit) gebracht worden. Während aber gegen letzteres Blatt kein Verfahren eingeleitet wurde, ist Leo Frankel unter der Anklage von Presseaufwiegelung sofort unter Anklage gestellt worden. Er wurde in erster Instanz für schuldig erklärt und zu zwei Jahren Kerkerstrafe verurteilt. Erst nach langem Hin und Her, in Dezember 1880 geriet die Berufung vor das schwurgerichtliche Pressegericht, welches die staatsanwaltliche Beweisführung für ungenügend erklärte und eine Nichtigkeitsbeschwerde anmeldete. Das höchste gerichtliche Forum, die Kurie, hingegen erklärte Leo Frankel unter Berufung auf die bereits erwähnte Presseverordnung vom Jahre 1867 und auf deren 171, 172. und 173. Paragraphen für schuldig, ohne ein neues Beweisverfahren angeordnet zu haben. Die Kurie erklärte das Ersuchen des Verteidigers, die beiden Zeitungsartikel miteinander zu identifizieren, für unberechtigt, billigte aber das Vorgehen des erstinstanzlichen Gerichts, dem Angeklagten die Einreichung einer Gesetzmässigkeitsbeschwerde verweigert $\mathrm{zu}$ haben, indem es den Auftritt des Angeklagten im voraus als ",nihilistische Provokation" bezeichnet hatte. Der Kurialbescheid sagt zum Schluss aus: der Umstand, dass , der Angeklagte nicht der Verfasser der Mitteilung ist, ändert nichts am Wesen der Sache, da nach dem - übrigens auch vom Strafgesetz beibehaltenen - 33. Paragraphen des Pressegesetzes nicht nur der Verfasser, sondern auch der Redakteur Verantwortung schuldet, und die Verantwortung sich auch auf jene Artikel erstreckt, die von anderen Blättern übernommen worden sind." Der Richter, der sich zu diesem Bescheid hingab, hiess Lörinc Tóth, ein Mann, der wegen seiner revolutionären Tätigkeit in den Jahren 1848-49 zurückgesetzt, später aber nach dem Ausgleich ,in Anerkennung seiner richterlichen Verdienste" auf leitenden Posten versetzt worden war. Er sandte die Sache ans erstinstanzliche Gericht zurück, indem er dessen ursprüngliches Verfahren als regelmässig bezeichnete. Daraufhin wurde Leo Frankel zu zwei Jahren Kerkerstrafe verurteilt. 